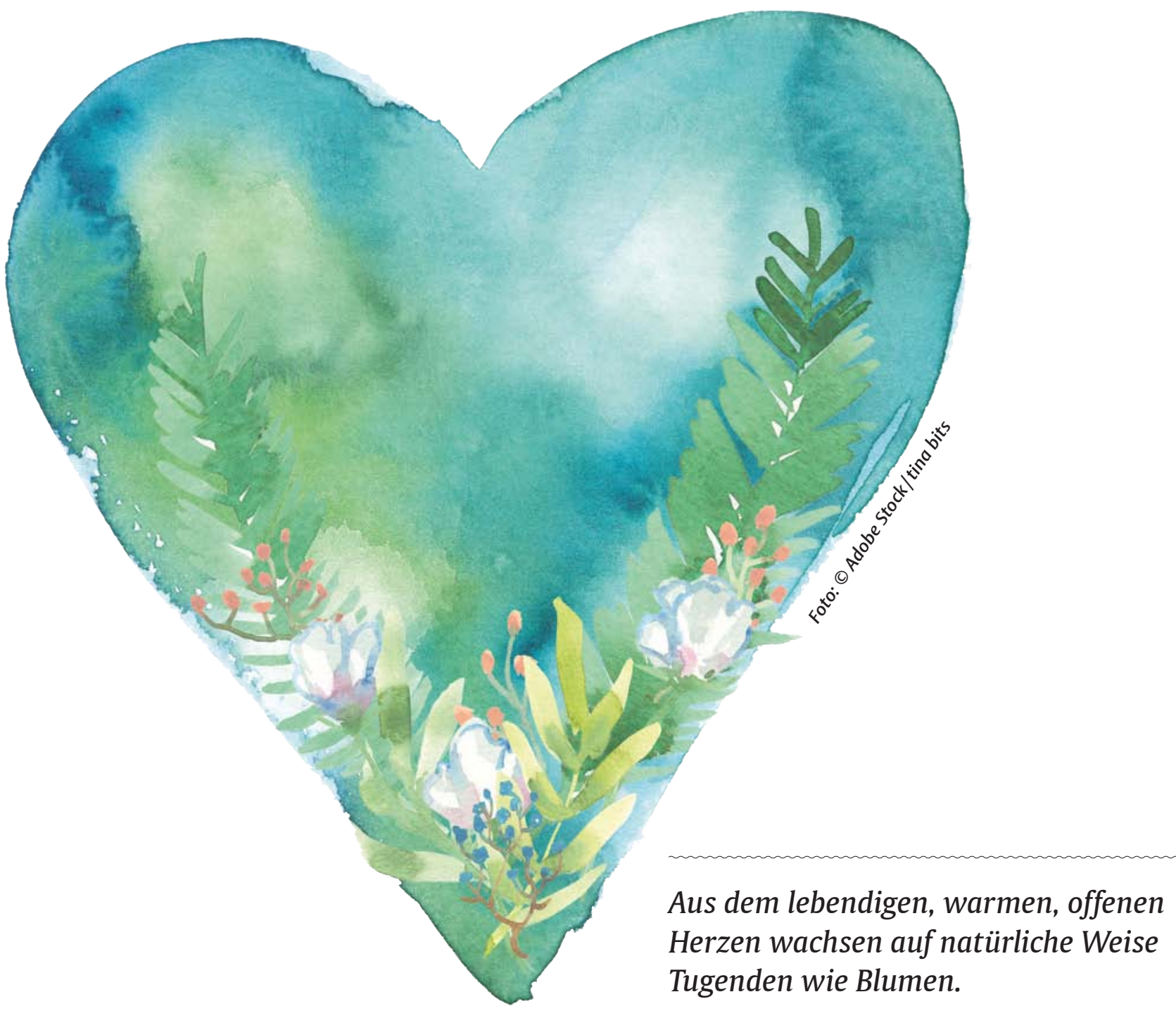

\title{
Das heilsame Herz
}

\section{Wohlwollen, Präsenz, Liebe: QUALITÄTEN DES HERZENS in der Therapie und wie wir sie entwickeln und verwirklichen können. Peter Findeisen}

Wir als Therapeuten sind mit existenziellen Fragen konfrontiert, mit dem Sinn von Leid, Tod, Suizidalität, Krankheit und massiven inneren psychischen Konflikten. Wir sind daher herausgefordert, neben einer guten Grundausbildung selbst eine innere heilsame Haltung zu all diesen Fragen zu finden, und dies im Laufe unseres Lebens immer wieder neu. Von uns sind Eigenschaften gefordert, als Lebenshaltung, als Charakterzug, die über ein therapeutisches Basiswissen hinausgehen und doch dazugehören. Die Rede ist von Herzensqualitäten (siehe Kasten).

\section{Das Herz ist Sinnbild}

Das Gehirn, für das wir uns dank der Neurowissenschaftler begeistern, sei, so Høystad [1], lediglich ein Faktum. Das Herz jedoch sei Faktum und Symbol zugleich und es beinhalte als Bild eine körperliche und eine seelische Seite. Das Herz kann Fähigkeiten und Qualitäten beherbergen und Gefühlsbarometer sein. Dies hat ihm seine mystische Dimension gegeben. Es wird in vielen Kulturen als feinfühligstes, als empfänglichstes aller Organe bezeichnet, als hörend und als Sitz der Emotionen und des Gewissens. Oft ver- 
mittelt das Herz zwischen dem Irdischen und dem Himmlischen. Das Herz ist sprachlich gesehen eine Metonymie, das heißt ein Bild, das als Teil eines komplexen Phänomens stellvertretend für dieses Phänomen steht. Der Begriff „Herz“ repräsentiert damit ein breites Spektrum von Bedeutungen - körperliche beziehungsweise medizinische oder neurokardiologische wie auch psychische, seelische oder spirituelle Phänomene.

Die vielfältigen Bedeutungen des Begriffs finden sich auch im psychotherapeutischen Kontext. Die Menschen, die zu uns in ihrer Not kommen, haben etwas auf dem Herzen. Etwas oder jemand hat ihnen vielleicht das Herz gebrochen. Schweren Herzens haben sie sich auf den Weg gemacht oder sind der Stimme ihres Herzens gefolgt. Ihr Herz ist sogar physiologisch messbar aus dem Rhythmus gekommen, wie wir aus der Psychosomatik und Neurokardiologie wissen. Therapeuten öffnen sich für verwundete Herzen und üben sich immer wieder bewusst und mit anteilnehmendem Herzen in Formen achtsamen Zuhörens und in Präsenz - kommen in Berührung mit ihrem eigenen verwundeten Herzen.

Høystad beschreibt die anteilnehmende, reaktionsfreudige Natur des Herzens folgendermaßen: „Das empfindsame, warme, offene und flexible Herz reagiert auf das, was im und um den Menschen herum geschieht. Es vermittelt die grundlegenden Emotionen: Liebe, Mitgefühl, Leidenschaft und Leid“ [1]. Womit er wichtige Qualitäten des Herzens bereits auf den Punkt gebracht hat.

\section{Das lebendige Herz liebt}

Im arabisch-persischen Raum gibt es ein interessantes Bild: aus dem lebendigen, warmen, offenen Herzen wachsen auf natürliche Weise Tugenden wie Blumen. Solche Tugenden wie Liebe und Wohlwollen. Ein anderer Gedanke ist: Das Herz selbst ist ein Samenbeet, aus dem die Pflanze, nämlich das Selbst, wächst - und Gefühle Gedanken und Handlungen sind dann die Früchte des Herzens, das sich so offenbart.

Das lebendige Herz wird durch Sympathie mit allem lebendig gehalten. Eine Sympathie, die man wiederum durch vertieftes Verstehen erlernt. Paracelsus denkt in derselben Weise und wird von Erich Fromm folgendermaßen zitiert: „aber wer versteht, der liebt - je mehr Erkenntnis einem Ding innewohnt, je größer ist die Liebe“ [2]. Das entspricht einer Erfahrung, die wir Therapeuten machen können - nämlich, dass wir über unsere alltägliche Arbeit wesentliche Herzensqualitäten entwickeln können, wenn wir mit der Zeit ein immer tieferes Verständnis gewinnen und unser Herz durch unsere Sympathie und unser Wohlwollen lebendig bleibt.

\section{Eine Herzensangelegenheit: Die therapeutische Beziehung}

Nicht selten machen es uns die Patienten schwer, sie zu lieben. Sofern wir es noch nicht verstanden haben, erinnern wir uns dann in Inter- und Supervisionen daran, dass der Patient sozusagen „keine Chance“ hat, solange er nicht vom Therapeuten geliebt wird, das heißt, mindestens grundsätzlich angenommen und idealerweise ins Herz geschlossen wird.

\section{IN DER PRAXIS}

\section{Welche Herzensqualitäten gibt es?}

Die Liste der Herzensqualitäten, die in der Psychotherapie Bedeutung haben, ist lang und kann nicht vollständig wiedergegeben werden. Es sind Qualitäten eines Herzens, das im Leben Bedeutung und Sinn, Erfüllung und Schönheit finden kann.

Das ... Herz

- achtsame

- berührte

- dankbare

- empfindsame

- freie

- großzügige

- humorvolle

- kreative

- lebendige

- mutige

- offene

- präsente

- starke

- versöhnliche

- warme

- zarte

Ein anderer Aspekt ist, dass Patienten uns im Laufe des Prozesses mehr und mehr erlauben, geliebt zu werden. Die therapeutische Liebe kann in diesem Sinne ein behutsamer oder manchmal auch ein schwieriger Annäherungsprozess an die Quelle der Liebe sein.

Für uns schwierige Situationen entstehen, wenn das Feuer der Liebe in uns entfacht wird oder wenn umgekehrt die Patienten uns heftig lieben und es nicht bei einer milden Übertragungsliebe bleibt. Dann sind Herzensqualitäten wie die der Ethik und Klarheit gefragt.

\section{Die Beziehung als Wirkfaktor}

Viele Forschungsergebnisse weisen darauf hin, dass die psychotherapeutische Beziehung unabhängig von der Methode das wesentliche Element der psychotherapeutischen Wirksamkeit ist.

Lambert und Barley [3] stellten zum Beispiel fest, dass $40 \%$ des Therapieerfolgs auf außertherapeutische Faktoren zurückzuführen sind. Betrachtet man nur die verbleibenden Faktoren, entfällt die Hälfte davon (also 30\%) auf die therapeutische Beziehung.

Um therapeutische Beziehungen aufzubauen, aufrechtzuerhalten und zu lösen braucht es erlernte Fertigkeiten, erfahrenes psychotherapeutisches Geschick und menschliche Eigenschaften sowie Haltungen, zu denen ich die Herzensqualitäten zählen möchte.

Einige Herzensqualitäten in der therapeutischen Beziehung Wie wir wissen, kann das Beziehungsangebot des Patienten sehr destruktive Elemente enthalten, und wir müssen bei unseren Pati- 
enten sehr häufig mit einem unsicheren Bindungsverhalten rechnen. Es wird von uns erwartet, dass wir auf solche Beziehungsangebote konstruktiv reagieren und dem etwas entgegensetzen. Wir erfüllen auch eine therapeutische Elternfunktion und begegnen Grundbedürfnissen nach Bindung, Orientierung und Kontrolle.

Ein schönes Beispiel für Herzensqualitäten, die im therapeutischen Beziehungsalltag selbstverständlich sind, finden wir in der OPD-2 (Operationalisierte Psychodynamische Diagnostik). Dort wird im Zusammenhang mit der Behandlung überwiegend strukturbedingter Störungen beschrieben, dass es besonders bei tiefergreifenderen Störungen darauf ankomme, die Probleme im Bereich der Bindung zu beachten und dann wörtlich: „Diese Ebene des Erlebens wird durch Wortinhalte meistens unvollkommen erreicht, so dass in solchen Fällen die Haltung und das Verhalten des Behandelnden, z. B. Verlässlichkeit, Festigkeit, Wohlwollen für die therapeutische Wirkung wichtiger sind als die Inhalte des Gesprächs“ [4].

Festigkeit und Verlässlichkeit allein - insbesondere Festigkeit allein - könnten möglicherweise als hartherzig oder herzlos erlebt werden, obwohl sie beide durchaus als Herzensqualitäten anzusehen sind. Gepaart mit Wohlwollen ist die Chance größer, dass diese Haltung es den Patienten erleichtert, Neues zu lernen. Das heißt, die alten ungünstigen, vielleicht angstbestimmten neuronalen Vernetzungen werden gehemmt, und es werden einmal mehr positive Erfahrungen ermöglicht, die neue neuronale Wege bahnen.

\section{Herzensqualitäten im Sinne der Ressourcenorientierung}

Das Gehirn muss zunächst in einen möglichst angstfreien Zustand gebracht werden, damit von dort aus eine Hemmung der negativ wirkenden Muster ausgehen kann. Wenn das, was im Gehirn eines Menschen positive Bedeutung hat, stark aktiviert wird und der Mensch sich in diesem Kontext mit seinen Problemen auseinandersetzt, dann wird unabhängig von alten Gedächtnisspuren etwas Neues aufgebaut, von dem eine positive Wirkung auf die Probleme ausgeht.

Luise Reddemann betont dies in ähnlicher Weise als Arbeitsbasis, indem sie sagt: „Probleme lassen sich leichter lösen, wenn jemand in guter Verfassung ist. Dazu ist es zunächst einmal wichtig, den Patientinnen zu helfen, dass sie ihren hohen Stresspegel senken können und dann dazu, dass sie zu ihrer Kraft finden. Im Grunde kann dabei jede Fähigkeit und jede Stärke, die jemand hat, als Ressource genutzt werden.“ [5]

Ziel der Therapie ist es unter anderem, dass sich Patienten über neue Erfahrungen stabilisieren, und es ist als ressourcenorientiertes Handeln zu verstehen, wenn wir dabei auf Qualitäten des Herzens im therapeutischen Kontext achten.

\section{Herzensqualitäten in der Intervention}

Herzensqualitäten können wir in der Arbeit mit den Patienten bewusst fördern, indem wir mit ihnen zum Beispiel Achtsamkeits-,
Imaginations-, Wahrnehmungs- oder Meditationsübungen machen. Im besten Fall durchzieht die Kommunikation von Herzensqualitäten unser ganzes Tun.

\section{Wie kommunizieren wir Qualitäten des Herzens?}

Zunächst machen wir uns als Therapeuten immer wieder unterschiedliche Herzensqualitäten bewusst. Hierzu können etwa Meditations- oder Besinnungsphasen hilfreich sein. Wir achten darauf, welchen Eindrücken wir uns aussetzen - das heißt nicht zu viel belastendem Material - und dass auch positive Erfahrungen ihren Raum erhalten.

\section{In Resonanz mit dem eigenen Herzensraum sein}

Als Therapeuten haben wir die Bereitschaft, uns selbst zur Verfügung zu stellen für die Menschen, die zu uns kommen, ein Herz für sie zu haben, in Resonanz zu kommen, in einen Bewusstseinszustand, der sich metaphorisch als „Raum“ bezeichnen lässt. Ein solcher Bewusstseinsraum kann als grenzenlos erlebt werden.

Eine Metapher für den Herzensraum ist das Bild des leeren Kelches, der gefüllt und wieder geleert werden kann und selbst unbeeinträchtigt bleibt, sodass ihm die Qualität der Reinheit und der Unabhängigkeit zugesprochen wird. Anstatt mit Flüssigkeiten kann er sich mit Licht füllen. Licht steht hier als Metapher für subtile Energie, subtile Emotion. Um sich der Realität des Lebendigen anzunähern, kann man sich den Kelch selbst vorstellen, als bestünde er aus Licht. Oder statt des Kelches ist seit Jahrhunderten die Metapher des Spiegels gebräuchlich, der Eindrücke widerspiegelt.

Diese Vorstellungsbilder beschreiben eine Fähigkeit, mit der wir uns in einem Raum orientieren können, der dynamische Grenzen hat. Ein Spiegel kann den Patienten, mit denen wir arbeiten, und uns durch Widerspiegeln eine Grenze geben. Um Orientierung und begrenzende Strukturen zu geben, sind in der Regel weitere Qualitäten wie Wärme, Akzeptanz, Klarheit und der Dia$\log$ in Worten erforderlich.

\section{Der Begriff der Präsenz und der des weiten Herzens}

Diese Begriffe entsprechen Erfahrungen, wie sie in Meditationsschulen überliefert wurden. Man ist sich dabei sowohl der personalen wie auch überpersönlichen Dimensionen bewusst, nimmt also gleichzeitig mehrere Bewusstseinsebenen wahr. Dies ist eine Form der Gegenwärtigkeit, in diesem Moment ganz wach und offen zu sein für das, was der Patient zeigt, was er nicht zeigt und was er zeigen könnte, wenn er seine ihm noch verborgenen Potenziale verwirklicht hätte.

Präsenz ist ein erweiterter Begriff von Achtsamkeit und bezieht sich ebenso auf allgemein menschlich zugängliche Bewusstseinszustände, wie das schon länger in der Psychotherapie angewandte Prinzip der Achtsamkeit.

\section{Den Zustand der Tiefe des Herzens}

Diesen Zustand könnte man folgendermaßen beschreiben: Eine Patientin ist an einem subtilen Gefühl angekommen. Es ist Stille. Die Therapeutin empfängt diese Qualität, welche die Patientin 
fühlt oder empfindet. Sie stört die Stille nicht, sie bleibt in Resonanz mit dieser Qualität, um die Patientin weiter im Erleben dieses Zustandes zu unterstützen. Die Patientin fühlt sich angekommen. In der Tiefe ihres Herzens.

In der Regel wird solch ein Erleben von den Patienten als heilsam oder heilend bezeichnet. Dies ist eine Situation, in denen Patienten die Erfahrung machen, dass es hinter all den Verletzungen in der Tiefe einen heilen Kern gibt, der wiederentdeckt wird. Im Therapieprozess kann dies einen wichtigen Wandlungsimpuls bewirken.

\section{Der Herzensblick}

Aus der Perspektive des Herzens kann es bedeutungsvoll für uns sein, dass die Patienten Augen finden, in denen sie sich am besten aufgehoben fühlen. In denen sie sich wertgeschätzt, geachtet und akzeptiert fühlen, gerade wenn er oder sie aus den früheren Erfahrungen heraus einen ganz anderen, ablehnenden Blick kennt. Wenn wir ihnen als Personen mit einer Art überpersönlicher Liebe begegnen, dann machen sie vielleicht die Erfahrung, dass sie geliebt sind - und nicht, dass sie nur dann geliebt werden, wenn sie dem Therapeuten oder dem lieben Gott alles recht machen. Sie erleben während der Therapie vielleicht andere Dimensionen, in denen sie sich bewusst werden, dass sie ein Kind des Lebens an sich sind und dass sie auf Heilung angelegt sind als ein Wesen, das ein Selbstheilungspotenzial in sich trägt.

Mit einer ressourcen- oder potenzialorientierten Einstellung können wir Selbstheilungskräfte oder im Menschen schlummernde Kräfte sehen, die wach werden oder geweckt werden wollen. Die Sufis zum Beispiel sagen, dass ein bestimmter Blick, für den sie den Begriff „Blick des Inayat“ (Nazar Inayat) haben, eine Resonanz beim Gegenüber auslöst, die dazu führt, dass er sich gesehen fühlt. Inayat bedeutet: Aufmerksamkeit, Interesse, Beachtung, Sorge, am Herzen liegen.

Der Herzensblick sieht das Unvollkommene, auch das Vollkommene, den Archetyp oder die Ganzheit im Unvollkommenen. Mit dem Herzen zu sehen, bedeutet, im Sinne der Liebesmystik den göttlichen Geliebten zu sehen, so wie der menschliche Liebende seine Geliebte anschaut. Der Blick, der liebt, verurteilt nicht. Er kann als heilend erfahren werden. Er bedeutet, dass einem etwas am Herzen liegt. Dafür ist nichts zu klein, nichts zu unwichtig. Er spiegelt eine Einstellung wider, die keine Gegenleistung erwartet.

Als Elternblick könnte der Herzensblick umschrieben werden mit: Wie schön, dass du da bist, wie wunderbar du bist und wie liebenswert. Oder: Das Leben ist doch das größte Wunder überhaupt.

\section{Das kreative Herz}

Es kann ein begeisternder Gedanke sein, mit Freude die eigene Person als Kunstwerk zu betrachten. Die Wertschätzung der Kunst und die Verpflichtung zum moralischen Handeln könnten neben der Liebe und der Arbeit als menschliche Ziele dem Leben Sinn verleihen, soweit eine Überlegung Otto Kernbergs [6]. Die Wertschätzung der Kunst in dem Sinne, sich selbst als Künstler für die Schaffung der eigenen Persönlichkeit zu sehen, an sich selbst als Kunstwerk bewusst eine neue Eigenschaft herauszubilden, ist 
eine Einstellung, die einer psychotherapeutischen Haltung des kreativen sich selbst Gestaltens entspricht. Dazu können drei Fragen gestellt werden:

- Was verlangt die Situation von mir?

- Wie möchte ich werden?

- Welche Eigenschaft entwickelt sich in mir unabhängig davon? Die Antwort auf die dritte Frage ist oft schwer zu finden und braucht die Wahrnehmung der intuitiven Stimme des Herzens. Wenn eine neue Qualität, zum Beispiel Liebe, Freude, Freiheit, Klarheit, deutlich ins Bewusstsein rückt, mag sie wie eine zarte Pflanze auftauchen. Auf diese Weise kann sie gepflegt und beschützt werden, statt dass sie ohne Fürsorge abstirbt. Übrigens kann die Antwort auf alle drei Fragen dieselbe Qualität beinhalten. Hilfreich ist die Beachtung der verschiedenen Ebenen des Verhaltens, des Fühlens, des Körperempfindens, des Denkens und der inneren Bilder, wie man das zum Beispiel aus der Ego-StateArbeit und der Traumatherapie kennt.

Das kreative Herz zu wecken, heißt auch, sich die Zukunft in allen Einzelheiten konkret vorzustellen: Wie könnte eine Situation aussehen und was würde sie von mir fordern, um sie zu bewältigen, welche Eigenschaften könnte ich in dieser Situation entwickeln? Wenn ich mich zum Beispiel für eine von zwei möglichen Eigenschaften entschiede, könnte ich gegenwärtig vielleicht diese Eigenschaft besser entwickeln als die andere?

\section{Das Herz weiß die Antwort}

Zum Abschluss möchte ich noch kurz darauf eingehen, wie wir Herzensqualitäten entwickeln können.

1. Sie sind schon da. Ich mache sie mir bewusst oder

2. die Qualitäten sind in Ansätzen vorhanden und ich wünsche, sie zu entwickeln oder

3. ich vermisse eine Qualität und sehne mich danach,

4. ich werde mir darüber klar, welche Qualität neu auftauchen will - dann folgt:

5. ich gebe ihr meine Wertschätzung,

6. ich sorge für sie und übernehme Verantwortung für sie,

7. ich stimme mein Herz ein, wie ein Musiker sein Instrument,

8. ich übe täglich.

9. Da ich nicht alle Qualitäten auf einmal entwickeln kann, entscheide ich mich für eine.

10. Mein Herz weiß die Antwort.

Dieser Artikel ist online zu finden:

http://dx.doi.org/10.1055/a-1092-6973
Literatur

[1] Ole Martin Høystad. Kulturgeschichte des Herzens: Von der Antike bis zur Gegenwart. Köln: Böhlau; 2006

[2] Fromm E. Die Kunst des Liebens. 62. Aufl. Berlin: Ullstein; 2005

[3] Lambert M, Barley D. Research summary on the therapeutic relationship and psychotherapy outcome. Psychotherapy 2002; 38: 357-361

[4] Arbeitskreis OPD. Operationalisierte Psychodynamische Diagnostik 2. Das Manual für Diagnostik und Therapieplanung. Bern: Huber; 2006

[5] Persönliche Mitteilung von Luise Reddemann

[6] Kernberg OF. Einige Überlegungen zum Verhältnis von Psychoanalyse und Religion. In: Bassler M (Hrsg.). Psychoanalyse und Religion. Stuttgart: Kohlhammer; 2000

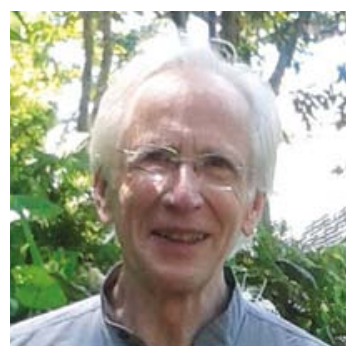

PETER FINDEISEN

Peter Findeisen ist Facharzt für Psychotherapeutische Medizin, Neurologie, Psychiatrie und Psychotherapie. Gemeinsam mit seiner Gattin Karin Püscher-Findeisen leitet er das Caduceus Zentrum, das unter anderem Gruppenretreats und Fortbildungen anbietet.

\section{E-Mail: zentrum@caduceus.de}

